\title{
Accuracy and clinical usefulness of scoliosis measurement with magnetic spine mapping - Ortelius
}

\author{
Zabka $\mathrm{M}^{1}$, Rehak $\mathrm{L}^{1}$, Uhrin $\mathrm{T}^{2}$ \\ 1st Department of Orthopaedics and Traumatology, Medical Faculty of Comenius University and \\ University Hospital Bratislava, Bratislava, Slovakia. martin_zabka@yahoo.com
}

\section{ABSTRACT}

OBJECTIVES: Our objective was to assess the accuracy of radiation-free magnetic method of scoliosis curve measurement (Ortelius 800) and its usefulness in clinical practice, as well as to judge whether it can replace $\mathrm{x}$-rays, which represent currently the golden standard for scoliosis measurement.

BACKGROUND: Patients with adolescent idiopathic scoliosis have to undergo multiple full-length spinal x-rays. Repeated exposure to ionizing radiation leads to higher incidence of breast cancer, thyroid cancer, and leukemia. One of the radiation-free methods that are candidates for replacing the x-rays is the magnetic measurement of the spine with Ortelius 800.

METHODS: Twenty-eight patients with adolescent idiopathic scoliosis were measured with Ortelius. Data from Ortelius were compared with standard spinal x-rays taken the same week.

RESULTS: The average Cobb's angle measured by $x$-ray was 35.11 degrees. The average absolute difference between Ortelius and $x$-rays was 6.69 degrees. The difference was statistically significant $(p=0.0443)$. An acceptable difference was measured in subgroup of curves that do not exceed 40 degrees (difference $5, p=0.8915$ ). CONCLUSION: Ortelius is most accurate in curves in range of 15-30 degrees of Cobb's angle. In larger curves it is increasingly inaccurate. Most important for clinical practice is the Cobb's angle range over 40 degrees, where the conservative therapy is to be replaced with surgery. Ortelius is not accurate enough in this range and therefore cannot fully substitute full spinal x-rays. It can be used to reduce the number of $x$-rays in the follow-up of smaller curve angles (Tab. 4, Fig. 8, Ref. 10). Text in PDF www.elis.sk.

KEY WORDS: adolescent idiopathic scoliosis, radiation-free assessment, Ortelius 800, magnetic spine mapping.

\section{Introduction}

Patients who have to undergo surgical treatment of scoliosis are followed with full-length spinal x-rays usually until they are 21 years old. The exposition to ionizing radiation takes place during the critical growth period, when the growing tissues of breasts, thyroid, bone growth plates, and other organs are susceptible to carcinogenic effects of radiation. The incidence of breast and thyroid carcinomas, as well as that of leukaemia is higher in patients who have undergone multiple spine $\mathrm{x}$-ray examinations $(1,2)$. In females who underwent $24 \mathrm{x}$-ray examinations on average, the risk of breast carcinoma is $70 \%$ higher than in normal population (1). Because only $10 \%$ of scoliosis patients develop curve progression that requires surgical treatment (3), it is appropriate to look for all possibilities for radiation-free scoliosis follow-up (4). One of the most recent methods is a radiation-free magnetic spine map-

${ }^{1} 1$ st Department of Orthopaedics and Traumatology, Medical Faculty of Comenius University and University Hospital Bratislava, Bratislava, Slovakia, and ${ }^{2}$ Department of Orthopaedics, Faculty Hospital Skalica, Skalica, Slovakia

Address for correspondence: M. Zabka, MD, 1st Department of Orthopaedics and Traumatology, Medical Faculty of Comenius University and University Hospital Bratislava, Ruzinovska 6, SK-826 06 Bratislava, Slovakia.

Phone: +421.2.48234724 ping using Ortelius 800 . The purpose of our study was to test the accuracy of scoliotic curve measurement compared to standard postero-anterior $\mathrm{x}$-rays and assess whether this method can replace $\mathrm{x}$-rays as a standard examination method for scoliosis follow-up.

\section{Materials and methods}

\section{Ortelius - magnetic spine mapping}

Ortelius 800 is a machine for simple measurement of the spinal deformities without exposing the patient to ionizing radiation (Fig. 1). It uses low-energy electromagnetic radiation to record the position of spinous processes of the vertebrae in space. After completing the examination, Ortelius automatically calculates the Cobb's angle, leg length discrepancy and other data about patient's balance that are important in pre- and postoperative follow-up of the patients.

\section{Examination process}

First, the patient bends forward and autoscoliometer connected to a fingertip scanner is slid down the patients back from top (spinous process of C7) to bottom (spinous process of S1) (Fig. 2), recording any asymmetry of the surface of the back (rib hump). According to this, Ortelius calculates the vertebral rotation in thoracic and lumbar segments of the spine.

In the next step, spinous processes are measured. The examiner uses a fingertip scanner on his forefinger and after he palpates 


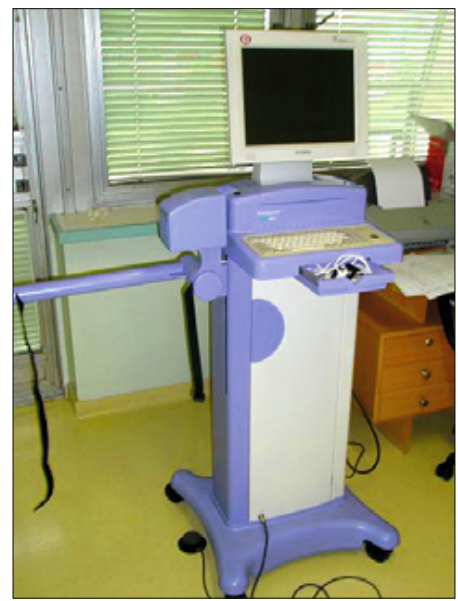

Fig. 1. Ortelius 800.

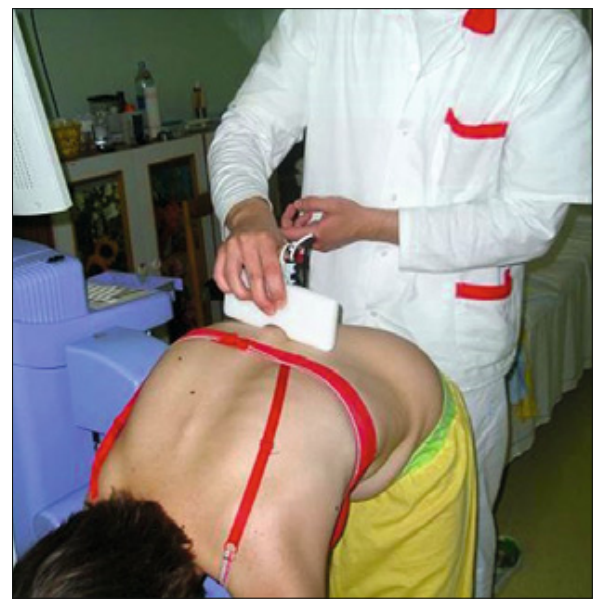

Fig. 2. Trunk rotation measurement with autoscoliometer.

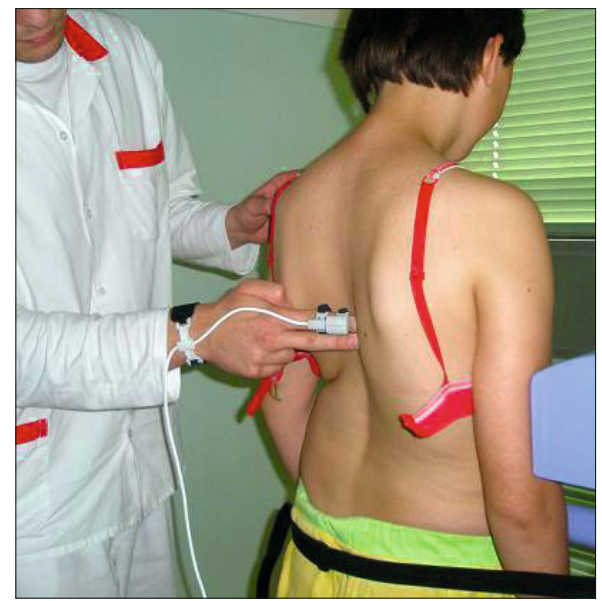

Fig. 3. Recording of spinous processes in space.

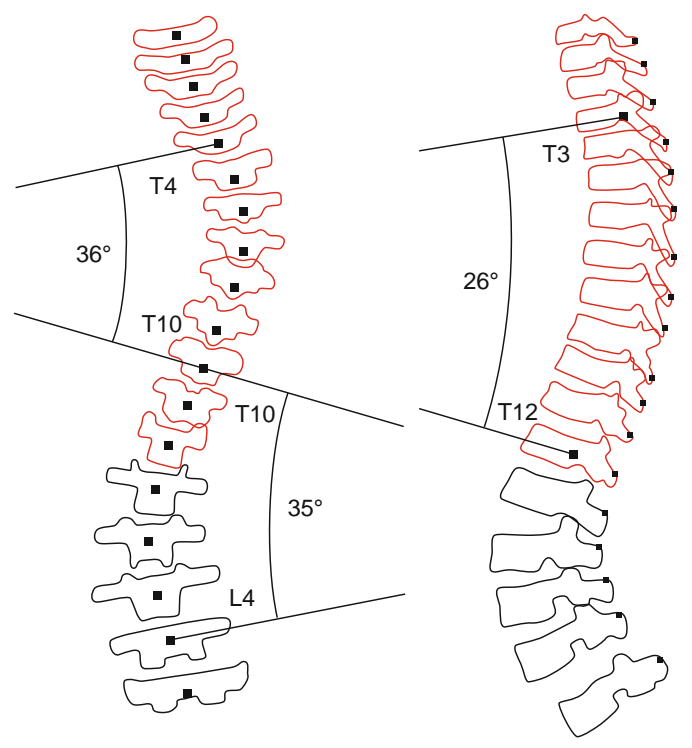

Fig. 4. Ortelius 800 output - PA and lateral view.
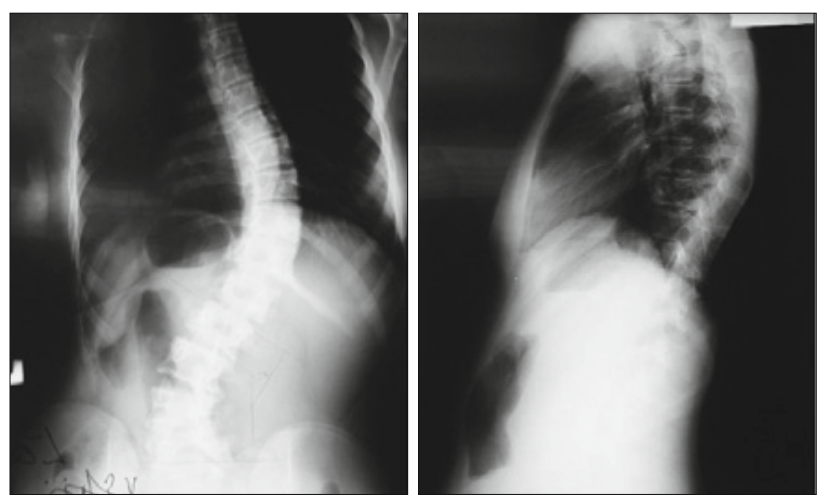

Fig. 5. X-rays of the same pateint.

the spinous process of each vertebra, he presses the footswitch to record its position in space (Fig. 3). The measurement is repeated once more to rule out the intraobserver error. Then the posterior superior iliac spines are palpated and marked. According to these data, Ortelius constructs a 3D picture of the spine, represented by frontal and sagittal images. The Cobb's angle is automatically calculated in frontal plane, while lordosis and kyphosis angles are in the sagittal plane (Fig. 4). Figure 5 shows $x$-rays of the same patient.

Digital x-ray pictures can be imported into Ortelius computer, while Cobb's angles are digitally measured and compared to Ortelius measurements.

In addition to spinal curve measurement, it is possible to measure patient's body balance (Fig. 6). Ortelius can record the position of the shoulders (acromioclavicular joints), pelvis (anterior and posterior superior iliac spines), femoral trochanters and mastoid processes on the head, thus calculating the possible pelvic asymmetry, shoulder height difference, leg length discrepancy and head tilt. All data are saved in a computer database,

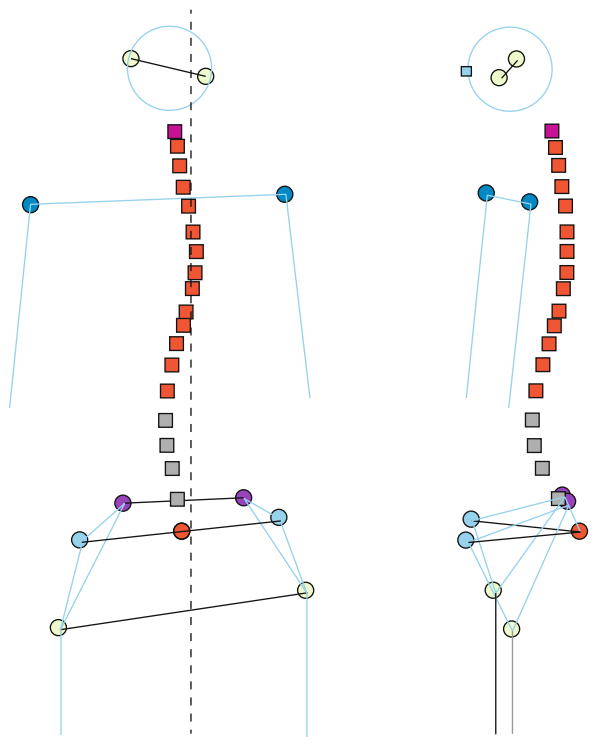

Fig. 6. Body balance data. 
to be able to measure the possible progression after repeated examinations.

At the 1st Department of Orthopaedics and Traumatology of University Hospital in Bratislava, we prospectively compared the findings of scoliosis examination with standard X-rays to Ortelius measurement. We examined 30 patients with adolescent idiopathic scoliosis (AIS) treated conservatively (follow-up or bracing, i.e. before surgical correction). The Cobb's angles measured on $\mathrm{X}$-rays of all patients did not exceed 55 degrees. Cobb's angles measurement on X-rays and Ortelius were taken during the same week. The examining position was altered according to Knott to increase the measurement accuracy (patient was touching the wall with his hands to help prevent the possibility of leaning forward, backward or to the sides). Measured values were statistically compared by paired t-test.

In two patients, it was not possible to palpate the spinal processes accurately because of subcutaneous fat tissue and pronounced lordosis. Because the recording of the positions of spinous processes was only approximate, the procedure could not be considered standard examination. As such it invariably led to inaccurate measurements, which were thus excluded from the study.

\section{Results}

\section{Results interpretation}

When interpreting the results of measurement by means of two different methods, one should take into consideration several ways of assessment. We can statistically average the exact values of Cobb's angle difference. In this instance, the results will show the difference in both positive and negative directions, i.e. if the angles measured by Ortelius are in general higher or lower than the x-rays (average difference). However these results can misinterpret the reality, e.g. in case of 5 positive and 5 negative values of 5 degrees in magnitude, the result would be 0 .

The second way is to average the absolute difference between $\mathrm{x}$-rays and Ortelius, (always positive value), which shows the accuracy of the method, but not the tendency of measuring greater or lower values. In the aforementioned example, the absolute average difference would be 5 .
Tab. 1. Cobb's angles measured on X-rays and Ortelius - aligned by increase in X-ray Cobb's angle.

\begin{tabular}{lccccc}
\hline X-ray & Ortelius & Difference & X-ray & Ortelius & Difference \\
\hline 12 & 14 & 2 & 30 & 27 & -3 \\
12 & 19 & 7 & 30 & 27 & -3 \\
15 & 28 & 13 & 36 & 35 & -1 \\
15 & 18 & 3 & 38 & 34 & -4 \\
15 & 25 & 10 & 39 & 27 & -12 \\
16 & 21 & 5 & 40 & 27 & -13 \\
17 & 19 & 2 & 43 & 36 & -7 \\
18 & 17 & -1 & 45 & 40 & -5 \\
22 & 27 & 5 & 45 & 30 & -10 \\
23 & 24 & 1 & 48 & 26 & -22 \\
23 & 27 & 4 & 50 & 46 & -4 \\
28 & 24 & -4 & 52 & 36 & -16 \\
29 & 24 & -5 & 52 & 40 & -12 \\
30 & 28 & -2 & 55 & 36 & -19 \\
\hline
\end{tabular}

With the paired T-test we can most accurately determine the correlation between the two methods, but we do not see the quantification of difference.

It is necessary to bear in mind that the results that are acceptable statistically do not have to be acceptable in clinical practice.

\section{Results}

In our study, we examined 28 patients; 27 girls and one boy (Tab. 1).

The average X-ray Cobb’s angle was 31.55 degrees (range 12-55 degrees). Thus we tested the whole examination range of Ortelius. The average difference between X-rays and Ortelius was -3.25 degrees (range $-22-13$ degrees). The average absolute difference was 6.69 degrees (range 1-22 degrees).

Our measurements were statistically tested by the paired T-test. The difference was statistically significant (two-tailed $\mathrm{P}$ value was 0.0443). 95\% confidence interval was $-7.47-0.10$; standard error of difference was 1.795. Summary is in Table 2.

While measuring the patients we noticed that the curves with high Cobb's angle value, especially those over 40 degrees, were measured less accurately with Ortelius. Curves larger than 55 degrees are actually listed in the manufacturer's exclusion criteria.

Tab. 2. Statistic results of group 1.

\begin{tabular}{|c|c|c|c|c|c|c|c|c|}
\hline$n=28$ & $\begin{array}{c}\text { Average } \\
\text { angle }\end{array}$ & Range & $\begin{array}{l}\text { Standard } \\
\text { deviation }\end{array}$ & Median & $\begin{array}{c}\text { Interquartile } \\
\text { range }\end{array}$ & $\begin{array}{c}\text { Paired Student's } \\
\text { t-test }\end{array}$ & $\begin{array}{c}\text { 95\% confidence } \\
\text { interval }\end{array}$ & $\begin{array}{c}\text { Standard error } \\
\text { of difference }\end{array}$ \\
\hline X-rays & 31.35 & $12 ; 55$ & 13.83 & 30 & $24 ; 34$ & $\mathrm{p}=0.0443$ & $(-7.47 ;-0.10)$ & 1.795 \\
\hline Ortelius & 27.92 & $14 ; 46$ & 7.66 & 28 & $17 ; 43$ & & & \\
\hline Average Difference & -3.25 & $-22 ; 13$ & 8.46 & -3 & $-10 ; 2$ & & & \\
\hline Absolute Difference & 6.96 & $1 ; 22$ & 5.68 & 5 & $3 ; 10$ & & & \\
\hline
\end{tabular}

Tab. 3. Statistics of subgroup 1 - up to 50 degrees.

\begin{tabular}{|c|c|c|c|c|c|c|c|c|}
\hline $\mathrm{n}=25$ & $\begin{array}{c}\text { Average } \\
\text { angle }\end{array}$ & Range & $\begin{array}{l}\text { Standard } \\
\text { deviation }\end{array}$ & Median & $\begin{array}{c}\text { Interquartile } \\
\text { range }\end{array}$ & $\begin{array}{c}\text { Paired Student's } \\
\text { t-test }\end{array}$ & $\begin{array}{l}\text { 95\% confidence } \\
\text { Interval }\end{array}$ & $\begin{array}{c}\text { Standard error } \\
\text { of difference }\end{array}$ \\
\hline X-ray & 28.76 & $12 ; 50$ & 12.22 & 28 & $16 ; 38$ & $\mathrm{p}=0.2269$ & $-5.22 ; 1.30$ & 1.581 \\
\hline Ortelius & 26.80 & $14 ; 46$ & 7.30 & 27 & $21 ; 28$ & & & \\
\hline Average Difference & -1.76 & $-22 ; 13$ & 7.61 & -3 & $-5 ; 2$ & & & \\
\hline Absolute Difference & 5.92 & $1 ; 22$ & 4.97 & 4 & $2 ; 7$ & & & \\
\hline
\end{tabular}


469-474

Tab. 4. Statistics of subgroup 2 - up to 40 degrees.

\begin{tabular}{|c|c|c|c|c|c|c|c|c|}
\hline$n=20$ & $\begin{array}{c}\text { Average } \\
\text { angle }\end{array}$ & Range & $\begin{array}{l}\text { Standard } \\
\text { deviation }\end{array}$ & Median & $\begin{array}{c}\text { Interquartile } \\
\text { range }\end{array}$ & $\begin{array}{c}\text { Paired Student's } \\
\text { t-test }\end{array}$ & $\begin{array}{c}\text { 95\% confidence } \\
\text { interval }\end{array}$ & $\begin{array}{c}\text { Standard error } \\
\text { of difference }\end{array}$ \\
\hline X-rays & 24.40 & $12 ; 40$ & 5.35 & 23 & $15 ; 30$ & $\mathrm{p}=0.8915$ & $-2.83 ; 3.23$ & 1.447 \\
\hline Ortelius & 24.60 & $14 ; 35$ & 9.33 & 25 & $19 ; 27$ & & & \\
\hline Average Difference & 0.2 & $-13 ; 13$ & 6.46 & -1 & $-4 ; 4$ & & & \\
\hline Absolute Difference & 5 & $1 ; 13$ & 3.94 & 4 & $2 ; 5$ & & & \\
\hline
\end{tabular}

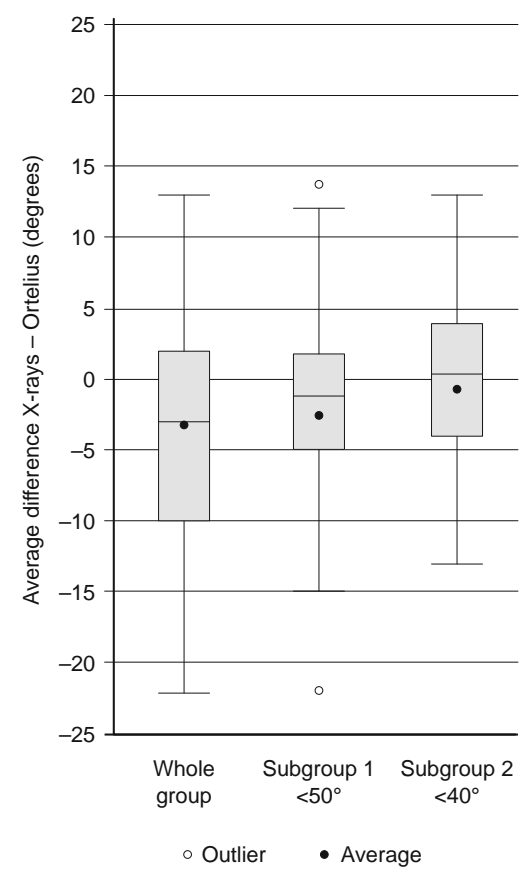

Fig. 8. Box-and-whisker plot.

To understand better the relation between curve magnitude and magnitude of difference, we assessed measurements in further two subgroups. From the original group we excluded curves above 50 (Subgroup 1 - up to 50 degrees) and above 40 degrees (subgroup 2 - up to 40 degrees).

In subgroup 1 (up to 50 degrees) (Tab. 3), the average absolute difference between x-rays and Ortelius was 6 degrees (standard deviation 4.97) and the average magnitude of curves measured was 28.76 degrees.

In subgroup 2 (up to 40 degrees) (Tab. 4), with the average curve measured being 24.4 degrees, the average absolute difference decreased to 5 degrees with standard deviation of 3.94 degrees. At the same time the maximum value of difference decreased from 22 to 13 degrees. The difference between Ortelius and x-rays in subgroups 1 and 2 was not statistically significant ( $p=0.22$ and 0.89 , respectively).

The average difference in the subgroups has a higher positive value when compared to group 1 ( -1.76 and 0.2 , respectively, as compared to -3.25 in the original group 1 ), which confirms a tendency towards negative values when measuring curves above 40 degrees. This tendency is obvious from Figure 7, where the paired values are sorted by the increase in X-ray Cobb’s angle.

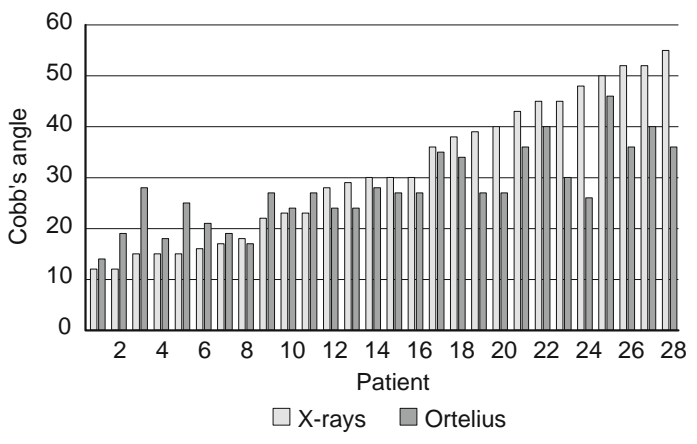

Fig. 7. Comparison of Cobb's angles measured on X-rays and Ortelius - aligned according to increase in $X$-ray value. It is obvious, that the best correlation between $x$-rays and Ortelius is in the range of 15-30 degrees. Angles smaller than 15 degrees were measured by Ortelius as bigger, angles above 40 degrees were measured by Ortelius as smaller.

Box-and-whisker plot of average difference between x-rays and Ortelius (Fig. 8) shows a tendency to measure positive or negative values and deviation. Boxes are the interquartile range. Both subgroups have more accurate results than the original group. Subgroup 1 (up to 50 degrees) has several outliers (i.e. a possibility of large difference). Subgroup 2 (up to 40 degrees) has an even distribution around zero.

\section{Discussion}

\section{Ortelius accuracy in literature}

The research of magnetic spine mapping in scoliosis patients has been focused on the preoperative follow-up. Dubousset et al (2003) (5) and Parsiny et al (2006) (6) tested the reliability of Ortelius 800 in scoliosis patients before surgical treatment and found out that when compared to standard x-rays, the difference in measurement was small. Ovadia et al (2007) found out that the average difference between X-rays and Ortelius was 5 degrees for Cobb's angle measurement and 6 degrees for lordosis and kyphosis measurements in the sagittal plane (7). Ortelius was also tested for accuracy in shoulder asymmetry and pelvic tilt measurement (8). However, Knott et al (9) pointed out higher differences and variability in multiple measurements, and pronounced this method to be inaccurate. In 2008 Knott himself proposed a new patient position during examination, which increased the measurement accuracy. The difference between Ortelius and X-rays decreased to 1.5 degrees on average, which are excellent results (10). Inter-observer and intra-observer variations of reliability were tested in several other studies $(7,9)$ where the measurements were consistent and without a 
statistically significant difference. According to literature, scoliosis measurement with Ortelius 800 can be considered accurate.

According to our results, the scoliosis assessment with Ortelius 800 cannot be considered reliably accurate. In conservatively treated patients with an average curve magnitude of 31 degrees, the Cobb's angle difference compared to x-rays is almost 7 degrees.

The logical explanation appears to be that Ortelius measures smaller curves more accurately. Figure 1 aligns the paired values by the increase in angle on x-rays. It shows that the best correlation is achieved in curves between 15 and 30 degrees. Curves smaller than 15 degrees are measured by Ortelius mostly as bigger, those over 40 degrees are measured as smaller. The biggest differences (4-22) were in curves between 40-55 degrees, where Ortelius is least accurate.

The absolute average difference of 7 degrees is higher by approximately 3 degrees than that in other published papers (4.40); however in those papers, the average curve measured on x-rays had only 17.8 degrees. These are almost the same results as in our postoperative group, where the average curve had 18.9 degrees and the average absolute difference was 4.5 degrees. Because of these findings, we took a closer look at the papers in question.

In the comments section to the study (7) there is a note, that if the initial difference between $\mathrm{x}$-rays and Ortelius was more than 10 degrees, it was not recommended to continue with Ortelius follow-up. In their results section, Ovidia et al (2007) published only the interquartile range of 2-7 degrees (3-10 in our study), but the histogram in the same paper tells that in $10 \%$ of cases the difference was 10-12 degrees, and several patients had the difference of 18 degrees. Knott et al (2006) published much worse results. In curves with the average magnitude of 29 degrees, the average difference was 8 degrees (comparable to our group). The maximum difference was 51 degrees; the big differences were measured mostly in curves over 40 degrees. We can conclude that although the average difference between $\mathrm{x}$-rays and Ortelius is 4 degrees, it can often amount to 7-10 degrees (25th-75th percentile), with several differences up to 20 degrees. It is the possibility of this error in both directions that can be a disadvantage in clinical practice.

During the examination we noticed, that curves over 40 degrees were measured inaccurately. Curves over 55 degrees do not even meet the inclusion criteria of the manufacturer. We suppose that this is caused by bigger vertebral rotation in more severe curves, where the curve of spinous processes recorded by Ortelius is smaller than the real curve of vertebral bodies on x-rays. The threshold of 55 degrees was probably the manufacturer's arbitrary decision, when the differences were not acceptable.

Thus we analysed the results in another two subgroups of the original group, where the results were comparable to other papers. From the original group we excluded patients with curves over 50 and over 40 degrees. In the subgroup up to 50 degrees, the average absolute difference was 6 degrees, and the average curve had 28.7 degrees. In the subgroup up to 40 degrees, the difference decreased to 5 degrees (average curve had 24.4 degrees), which is comparable to other papers. At the same time the maximal differ- ence measured decreased from 22 to 13 degrees. Figure 8 shows the histogram of all three groups; subgroup 2 (up to 40 degrees) has the most even distribution around zero.

This proves that Ortelius measurements are most accurate for curves from 15 to 30 degrees.

\section{Usefulness of Ortelius 800 in clinical practice}

Indication criteria of Ortelius define the possible range of examinable curves from 10 to 55 degrees. The spinal curve is considered to be scoliotic when greater than 10 degrees; the upper limit was probably chosen arbitrarily because of greater inaccuracies in bigger curves. In our study we have found out that accurate measurements applicable to clinical practice can be achieved in curves from 15 to 30 degrees. These patients undergo conservative therapy (bracing or follow-up). Criteria for changing the conservative treatment to surgery are met when the curve has 40-50 degrees with progression, in this range however, Ortelius is less accurate with a tendency to measure lower values (in extreme cases even 10-20 degrees).

This is the reason why we do not recommend using Ortelius in this range of curve magnitude. The patient can have a curve that is indicated for surgical correction, but Ortelius would measure only a curve that is less than 40 degrees. It is suitable for following the patients treated conservatively with or without a brace, i.e. in cases where it can be a substitution for several, but not all x-ray examinations. One way to go is to order an $\mathrm{x}$-ray if Ortelius shows 30 degrees or more. Another possibility is to take x-rays once a year, and in the meanwhile follow the patients only with Ortelius. Thus we could cut down on the number of x-rays and significantly decrease radiation exposure of the patient.

Ortelius has good correlation with x-rays in small curves up to 30 degrees, but there is a rare possibility of the difference up to \pm 13 degrees. We can measure a smaller instead of a greater curve and miss the progression (with all disadvantages of late surgical treatment), or measure a greater curve instead of a smaller one (although in this case, we can still confirm the progression with $\mathrm{x}$-rays). It is up to the orthopaedist whether he is ready to accept this slight risk of false measurement.

We can conclude that Ortelius cannot replace long spinal $\mathrm{x}$ rays in scoliosis diagnostics and follow-up of curve progression towards surgical treatment. However, it can be used to decrease the number of $\mathrm{x}$-rays, especially during the conservative phase of treatment.

\section{Learning points}

Ortelius 800 uses magnetic field to measure the position of spinous processes in space and thus the shape of the scoliotic curve.

It is a radiation-free measurement.

It is accurate enough for clinical use in spinal curves with Cobb’s angle from 15 to 30 degrees.

In curves over 40 degrees it becomes unpredictibly innacurate.

Full-length spinal x-rays remain the golden standard in scoliosis assessment. 
469-474

\section{References}

1. Doody M, Lonstein JE, Stovall M, Hacker DG, Luckyanov N, Land CE. Breast cancer mortality after diagnostic radiography: findings from the U.S. Scoliosis Cohort Study. Spine 2000; 25: 2052-2063.

2. Rao PS, Gregg EC. A revised estimate of the risk of carcinogenesis from X-rays to scoliosis patients. Invest Radiol 1984; 19: 58-60.

3. Miller NH. Cause and natural history of adolescent idiopathic scoliosis. Orthop Clin North Am 1999; 30: 343-352.

4. Ovadia D, Dickman D, Fragniere B, Leitner J, Rigo M, Dubousset J. A Non-Invasive Approach for Scoliosis Assessment. Oral presentation, EuroSpine, October 2003. Prague, Czech Republic.

5. Dubousset J, Ovadia D, Leitner J, Fragniere B, Rigo M, Dickman D. A non-invasive approach for scoliosis assessment. Spine 2003; 3 (Suppl 5): 156-157.

6. Parisini P, Lolli F, Greggi T, Di Silvestre M, Cioni A, Giacomini S, Bakaloudis G. An innovative diagnostic procedure of vertebral deformities without exposure to X-rays. Stud Health Technol Inform 2006; 123: 527-532.
7. Ovadia D, Bar-On E, Fragniere B, Rigo M, Dickman D, Leitner J, Wientroub S, Dubousset J. Radiation-free quantitative assessment of scoliosis: a multi center prospective study. Eur Spine J 2007; 16: 97-105.

8. Parisini P, Di Silvestre M, Greggi T, Cioni A, Giacomini S, Bakaloudis G, Lolli F: Innovative Diagnostic Procedure, with 3D Reconstruction of Vertebral Deformities Without Exposure to X-rays, Eur Spine J 2005;14 (Suppl 1): S24.

9. Knott P, Mardjetko S, Nance D, Dunn M. Electromagnetic Topographical Technique of Curve Evaluation for Adolescent Idiopathic Scoliosis. Spine 2006; 31 (24): E911-E915.

10. Knott P, Mardjetko S, Dunn M, Johnson D, Cruze S, Moore J. Evaluating the Influence of Patient Positioning on the Accuracy of Ortelius 800 Measurements for Scoliosis. Abstract 5th International Conference on Conservative Management of Spinal Deformities Athens, April 3 - 52008. In Scoliosis 2009; 4 (Suppl 1): O18.

Received July 7, 2014. Accepted March 8, 2015. 